\title{
Immediate Restorations on Implants in the
} Esthetic Area

\author{
${ }^{1}$ Pedro Peña Martinez, ${ }^{2}$ Ramón Palomero Langner, ${ }^{3}$ Ramón Palomero Rodríguez \\ ${ }^{1}$ Doctor of Medical Dentistry, Oral Surgeon, Director of European Forum of Implantology, Madrid, Spain \\ ${ }^{2}$ Postgraduate in Prosthetic Implantology, European Forum of Implantology, Madrid, Spain \\ ${ }^{3}$ Doctor in Stomotology, Specialist in Maxillo-Facial Surgery, Former Chief of Hospital Services, Virgen del Camino (Pamplona)
}

Correspondence: Pedro Peña Martinez, Doctor of Medical Dentistry, Oral Surgeon, Director of European Forum of Implantology C/Caracas No-5, Local 28010, Madrid, Spain, e-mail: forum_implantologico_europco@yahoo.es

\begin{abstract}
Implant placement and restoration in the esthetic zone is a complex treatment modality, in which many factors are important to achieve success. Extraction defects are treatable with immediate implantation with predictable results.
\end{abstract}

Keywords: Immediate implant, immediate restoration, extraction defects.

\section{INTRODUCTION}

\section{Science and Practice}

The placement of the implants in the alveolus postextraction has become a routine procedure the last few years and it has strengthened as the elected procedure in the postextraction defects type 1 (Hammerle et al, ITI Consensus). In these cases we are not talking about "immediate load" but "immediate provisionalization", a term that implies the realization of a prothesis within a 48 hours time frame after the placement of the implant. This combined with the concept of immediate implant postextraction, implies the placement of an implant in the alveolus postextraction and becoming part of the same surgical procedure.

Thus far the procedure has not been validated enough by the scientific community but the number of studies that are taking place are showing a series of considerations that we should take into account when performing this kind of treatment:

- It seems that one of the fundamental conditions that should exist is the proof that the implant that is placed in the postextraction alveolus has the appropriate "primary stability". This requires that we relocate the apex of the implant to the palatine bone and extend at least 3 to $5 \mathrm{~mm}$ further past the location of the root apex.

- There is some controversy on whether it is recommended to place a graft in the 1 and $2 \mathrm{~mm}$ gaps that will remain between the implant and the vestibular bone of the patient. More questionable should be wether to treat with membranes or simply with the clot that will form after the placement of the implant.

- Some authors commend and have demonstrated histologically the benefits of immediately loading the provisional prothesis placed over these implants and this can make us doubt when the time to decide to load or not our provisional prothesis, even more when it is of unitary teeth in the esthetic area.

\section{MATERIALS AND METHODS}

In the following section we will describe the surgical/ prosthetic procedures used to treat all of our patients that have the Type 1 defect in the esthetic area. These situations require the need for extracting a full tooth and/or root in the esthetic area (canine to canine); the substitution of the root with a titanium implant functioning as the foundation for a provisional restoration.

Utilization of the procedure, is referred to as "immediate restoration".

A fundamental part in the surgical technique requires the need to extract the full tooth and/or root while incurring minimal injury to the surrounding hard and soft support tissues. In many occasions the extraction becomes the most complicated part of the intervention. Some teeth suffer horizontal fractures or cavities that prevent the routine use of forceps. These defects will require the use of sindesmotomos, electric scalpel (Mectron), pressure separators (X-tract) or turbine with dental section components (Zekrya, Mailleffer). 
The placement of the implant starts by making a slot in the apical third of the palatine wall of the alveolus. The slot ensures we will have a strong starting point preventing the drill from sliding through the alveolus hard plate to the old location of the dental root.

Depending on the perception of the bone density during the "site preparation" which must extend at least $3 \mathrm{~mm}$ above the dental root location, we will select the implant design that will adapt best to it. There is an industry consensus that conical implants with the shape of a root are the most suitable for this procedure (RePlant ${ }^{\mathrm{TM}}$, Implant Direct LLC), recently implants with a specific thread design have become available (ReActive ${ }^{\mathrm{TM}}$ or Legacy3, Implant Direct LLC) that enable you to achieve a higher insertion torque through the infra preparation of the osteotomy that supposedly is implemented in the last step of the "site preparation" sequence.

Once the implant is placed in the desired tridimensional desired position, we graft the existent space between the implant and the vestibular wall of the alveolus with a
"Xenoplast (Bio-Oss ${ }^{\circledR}$, Inibsa, Geistlich), whatever the dimensions and depth.

Following the above procedure we will make the provisional restoration on a titanium abutment or especially prepared plastic (Peek) abutment. This "custom" provisional abutment will provide the proper support to the "papillas" and the gingival margin and at the same time will offer esthetics and comfort to the patient.

\section{CLINICAL CASE}

A patient presented them self to our implant center with a referral for extraction and substitution with implants, tooth numbers, 11, 12, and 21 (Figs 1 to 2B). After the diagnosis, we decided to work on the implants in different stages starting with the tooth that presented the worst prognostic in the mouth.

We decided to extract the right central incisor (11) and substitute with a root shape conical implant (Replant Implant Direct LLC) with a trilobed internal connection and a dimension of $5 \mathrm{mmD} \times 13 \mathrm{mmL}$ (Figs 3 to 8 ).

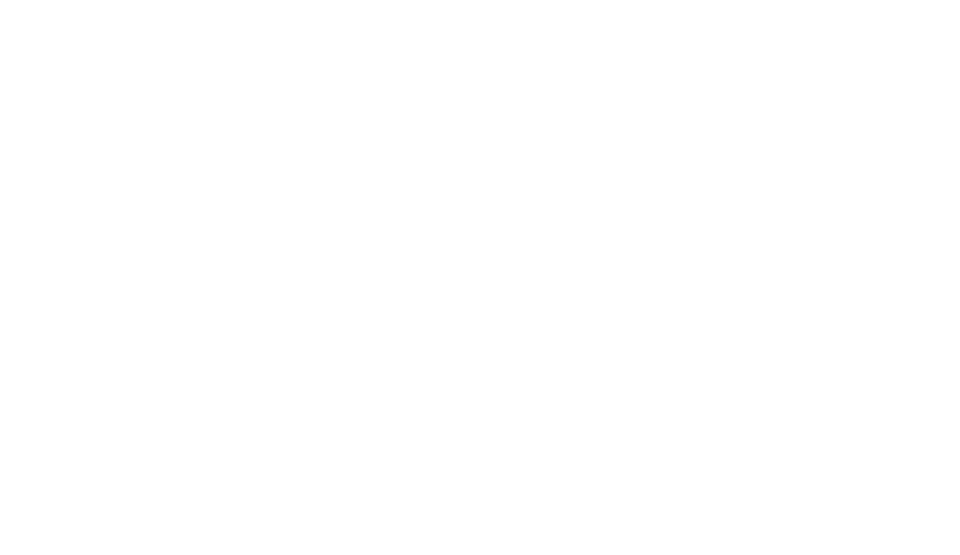

Fig. 1: Radiographic representation prior to the treatment with implants. The teeth 11, 12, and 21 presented fractures or cavities which makes them not viable for restoration with a secure prothesis
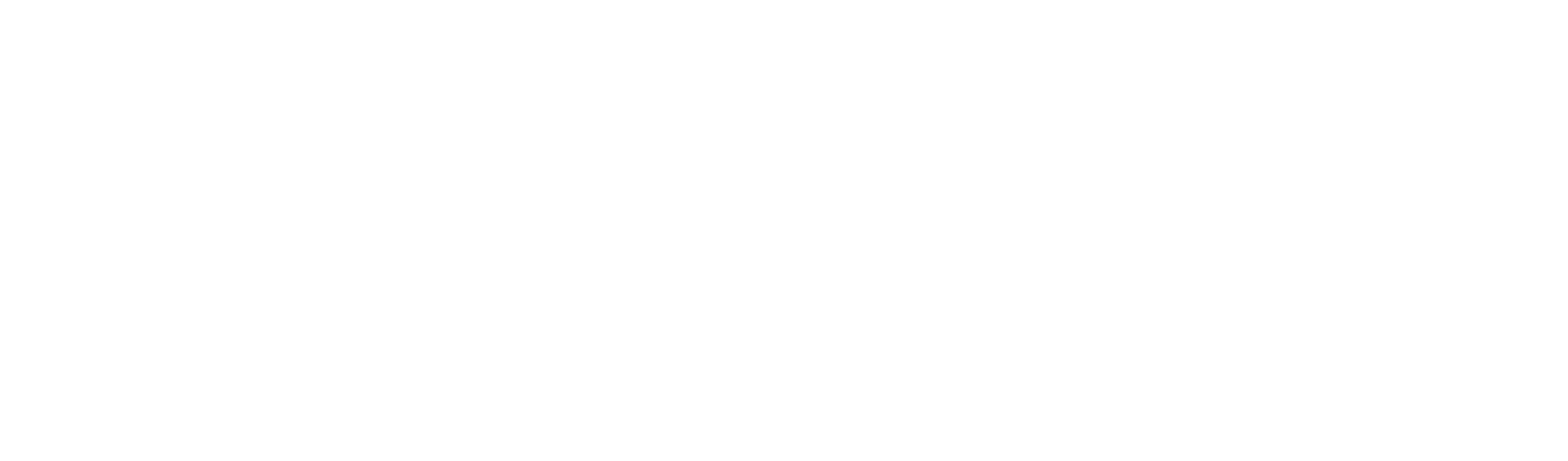

Figs 2A and B: Intraoral appearance of the patient. The right upper central incisor is a composite crown attached to the root without any retention 

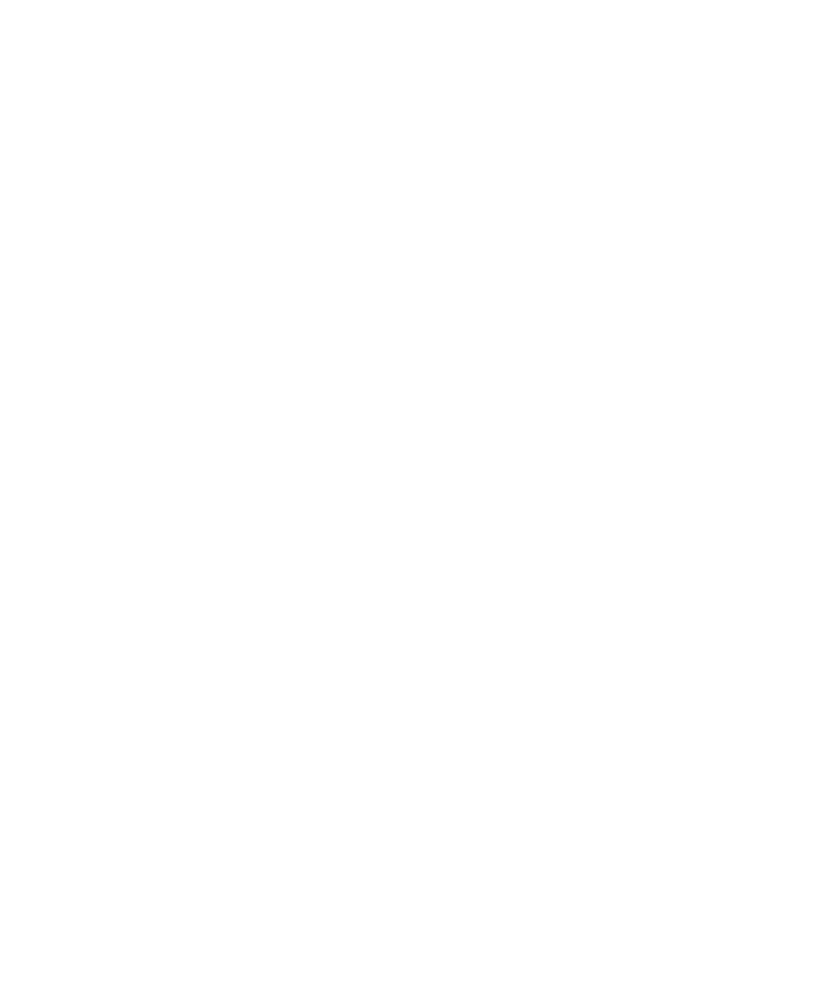

Figs $\mathbf{3 A}$ and $\mathbf{B}$ : The extraction must be done with extreme care, without lifting a flap and without damaging the surrounding tissue

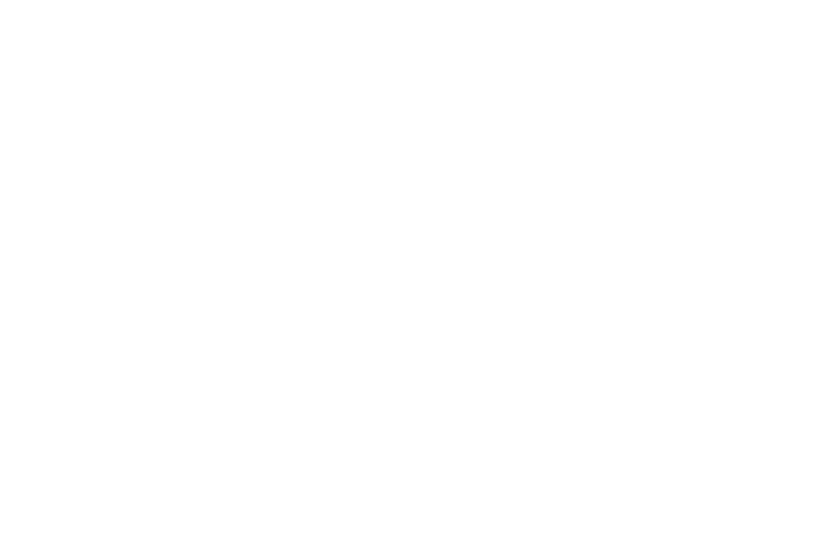

Fig. 4: Occlusal view of trilobe implant in place

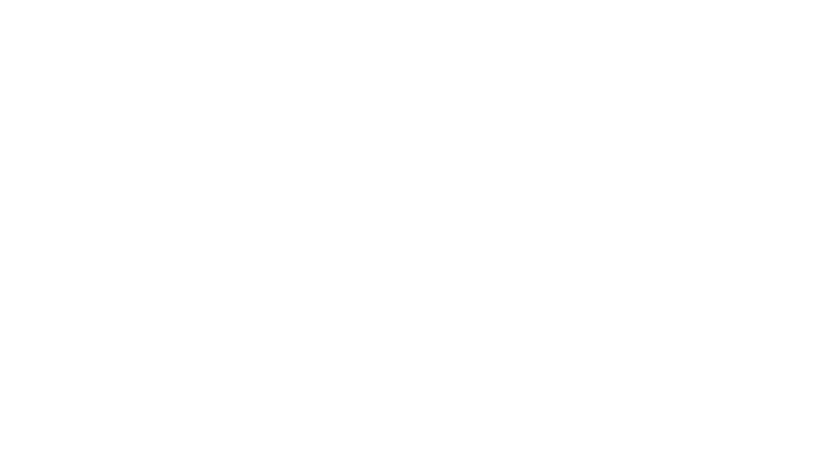

Fig. 5A

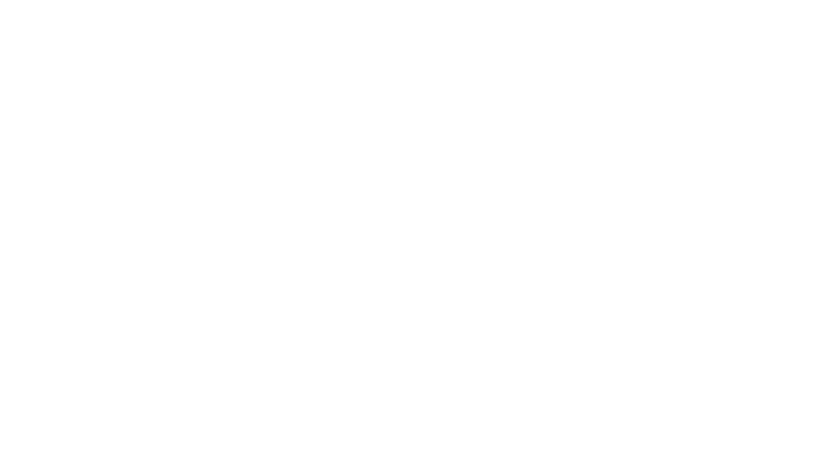

Figs $\mathbf{5 A}$ and $\mathbf{B}$ : Once the implant is placed, the Peek abutment is selected and seated on the implant to view proposed reduction enabling the design and placement of the provisional prosthesis

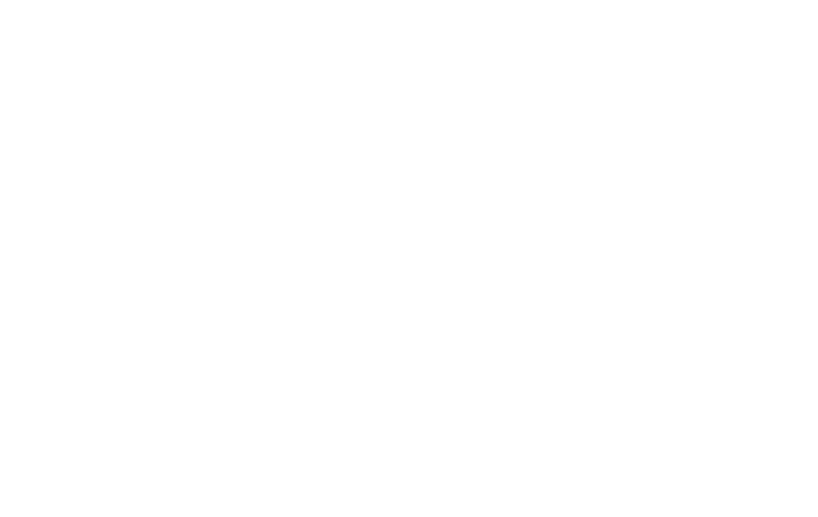

Fig. 6: The space between the implant and the vestibular wall of the alveolus is filled-up with Xenograft

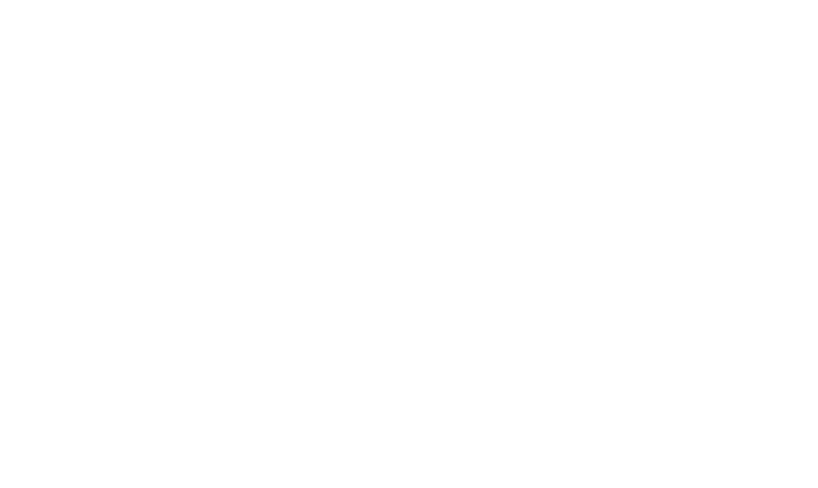

Fig. 7: The crown is cemented with a provisional cement without eugenol and it is kept out of all occlusal contact

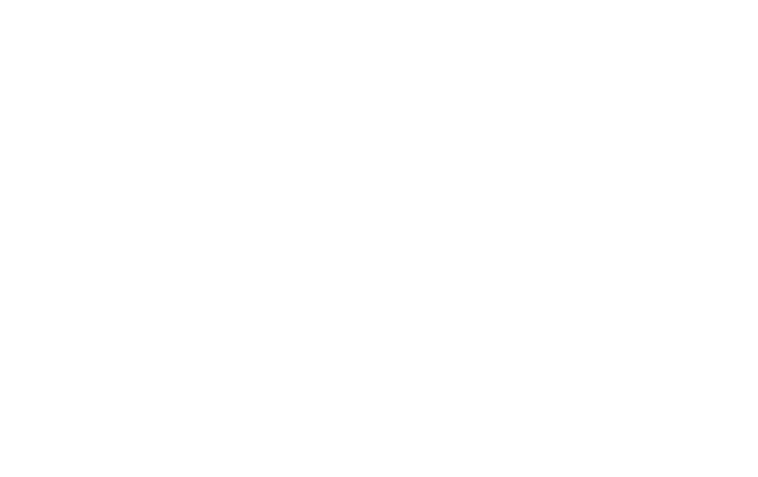

Fig. 8: X-ray two months after the implantation of the Replant ${ }^{\mathrm{TM}} 5.0 \mathrm{mmD}$ implant 
After two months of healing we proceeded with the extraction and substitution of the upper left central incisor (Figs 9A and B). Due to the presence of a bone with a lower bone density which was established during the early stages of the drilling procedure, we decided to place an implant with modified helix, high cut and internal hexagonal connection (Legacy3 Implant Direct LLC) of $4.7 \mathrm{mmD} \times$
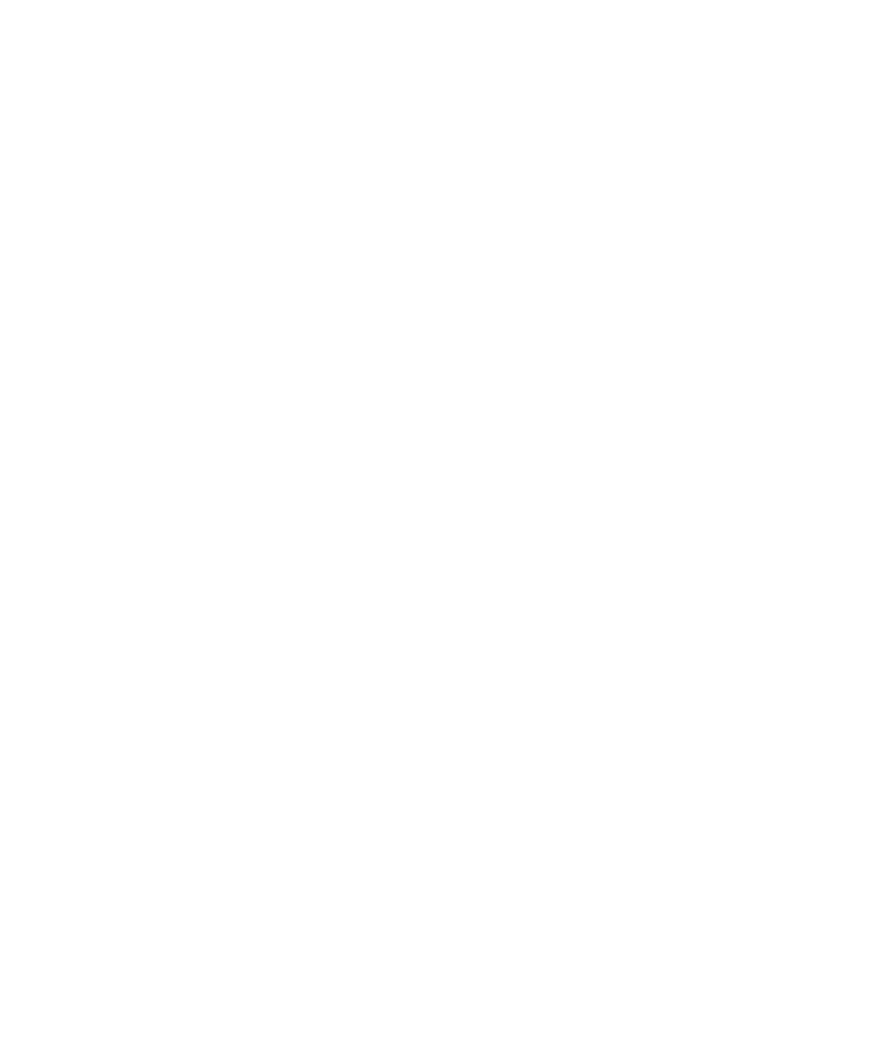

Figs 9A and B: Intraoral appearance after two months of healing. We proceeded to atraumatically extract the left upper central incisor

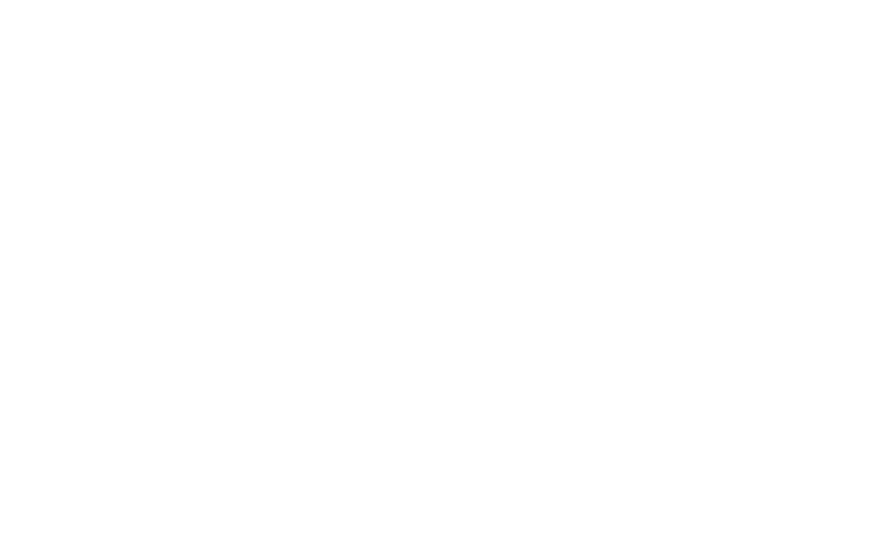

Fig. 10: Placement of Legacy3 implant which incorporates the modified thread pattern enabling higher insertion torque through the under preparation technique of the alveolus layer (insertion torque $>45$ $\mathrm{N} / \mathrm{cm})$
$13 \mathrm{mmL}$ dimensions (Figs 9A to 14B). The implant design enables us to reach the primary stability desired to be able to proceed with the immediate prothesis.

In both cases we grafted the GAP with a partially resorbable Xenograft (Bio-Oss ${ }^{\circledR}$, Geistlich) and the prothesis made chair side were placed providing assurance that there were not any kind of contact either in the centric nor in the lateral or protrusive excursions.

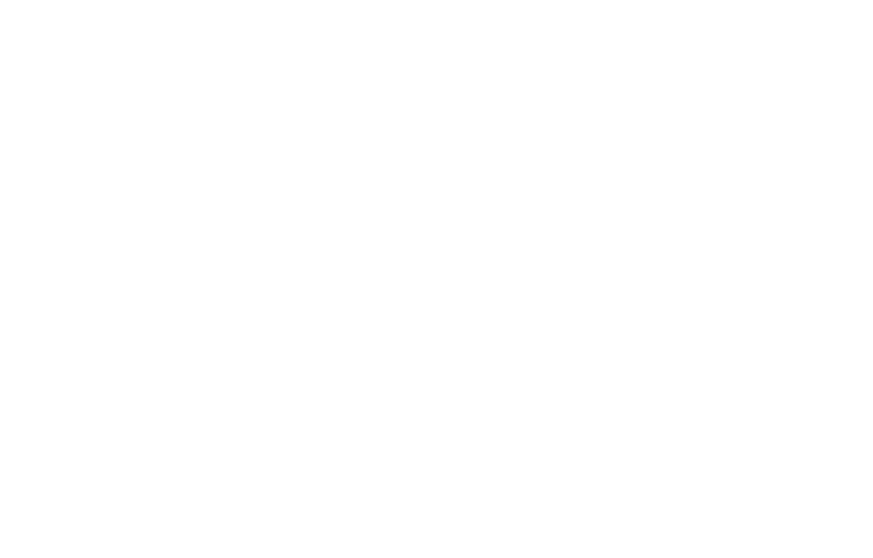

Fig. 11: Peek abutment modified to house the provisional crown

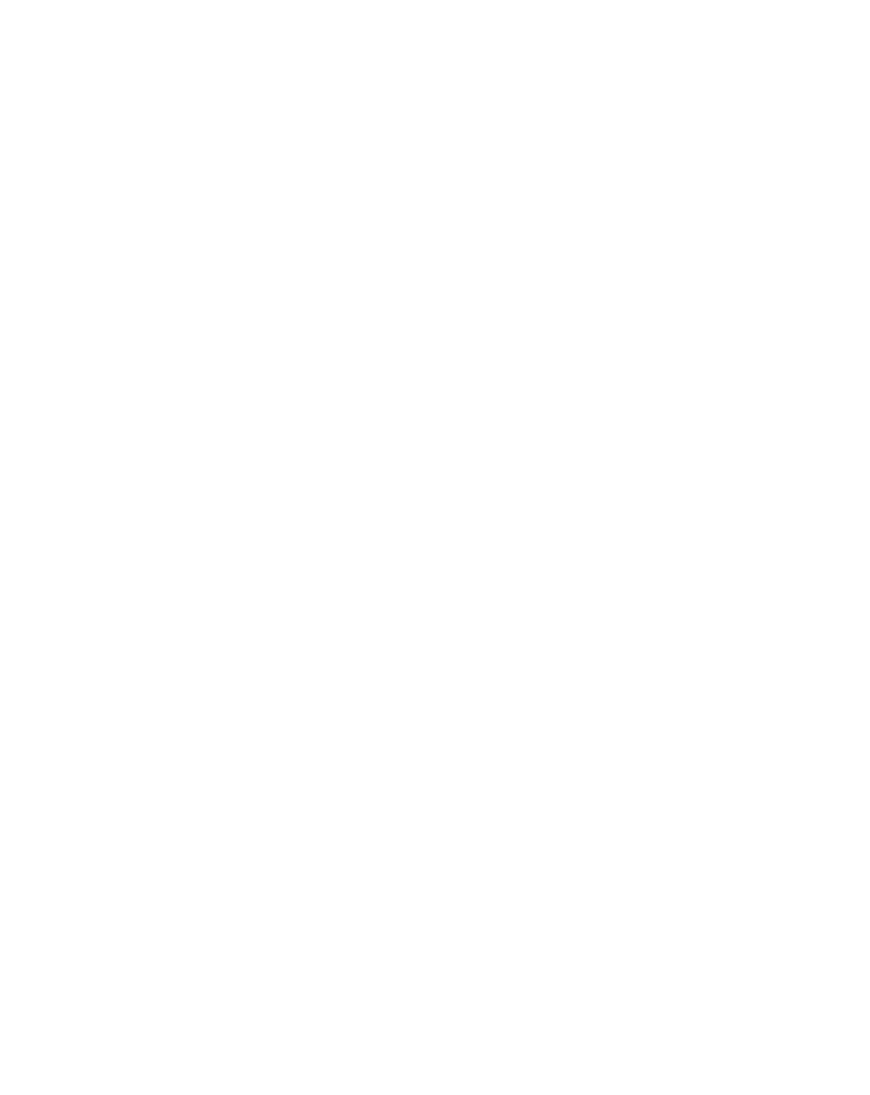

Figs 12A and B: Abutment and crown joined and the profile of the subgingival one third is back-filled with a composite material to fill in the gap to the proposed clinical crown margin 


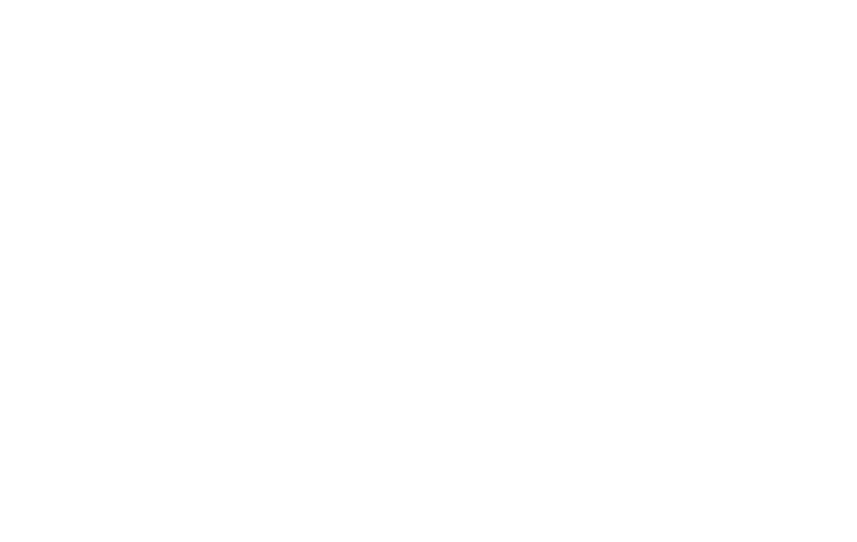

Figs 13: Appearance of the two provisional crowns after being cemented
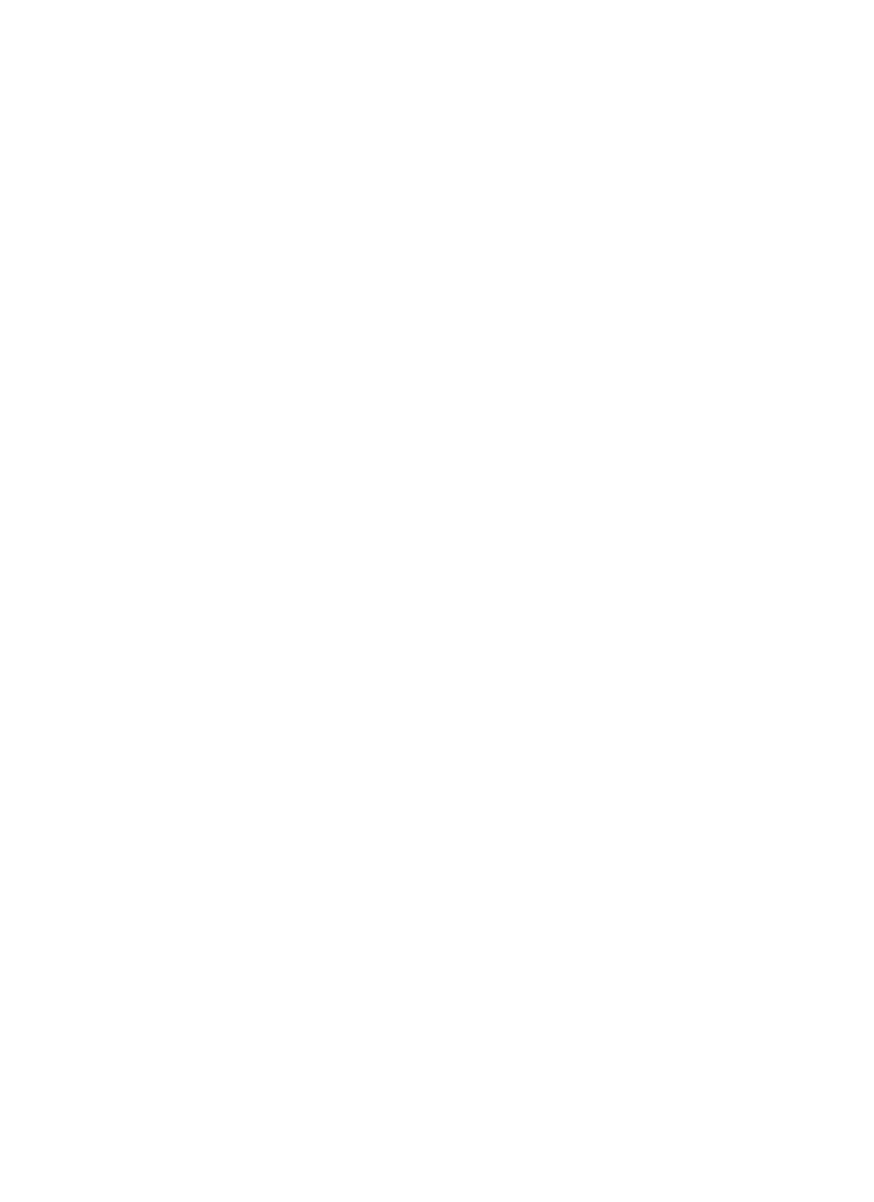

Figs 14A and B: Early healing. Appearance five months after the placement of the central right incisive and three months after the placement of the central left incisive

\section{DISCUSSION}

Now we know the remodeling processes of the postextraction alveolus and the loss of volume that endure. Of course, these processes depend on the elapsed time since the extraction, the presence or not of previous pathology and the type of prosthesis that the patient has had during the time that stood toothless.

Literature seems to endorse the positive result of this type of treatment. The combination of an atraumatic extraction with an implant placement immediately after the postextraction, leaves sufficient space to the alveolar table $(2 \mathrm{~mm})$. The later filling of said space with a partially resorbable biomaterial (Bio-Oss ${ }^{\circledR}$ ) and the placement of a provisional prosthesis, give support to the gingival architecture allowing it to maintain the esthetics and function of the patient.

These procedures often achieve implant-supported restorations undistinguished from a natural tooth as much as shape, position and gingival architecture.

\section{CONCLUSIONS}

The use of this protocol in our hands adequately allows us to treat the defects of postextraction Type 1, with immediate postextraction implants, graft the GAP and a provisional prosthesis without showing in the long run, the changes made in the controls.

\section{BIBLIOGRAPHY}

1. Immediate loading and flapless, postextraction, single-tooth implant restoration: Advantages and indications. Fabbri G, Ban G, Mancini R. Pract Proced Aesthet Dent 2008;20(10): 633-39.

2. Immediate implant placement postextraction without flap elevation. Chen ST, Darby IB, Reynolds EC, Clement JG. J Periodontol 2009;80(1):163-72.

3. Single-tooth replacement in the anterior maxilla by means of immediate implantation and provisionalization: A review. De Rouck T, Collys K, Cosyn J. Int J Oral Maxillofac Implants. 2008;23(5):897-904.

4. How does the timing of implant placement to extraction affect outcome? Quirynen M, Van Assche N, Botticelli D, Berglundh T. Int J Oral Maxillofac Implants 2007;22 Suppl:203-23. 